384 QUANTIFICATION OF IGM AND IGA ANTI-PNEUMOCOCCAL POLYSACCHARIDES BY A NEW ELISA ASSAY. A VALUABLE DIAGNOSTIC AND PROGNOSTIC TOOL FOR HYPOGAMIMAGLOBULINEMIAS

doi:10.1136/archdischild-2012-302724.0384

'I Quinti, 'FM Cavaliere, ' $\mathrm{C}$ Milito, 'G Brunetti, ${ }^{2} \mathrm{~K}$ Warnatz, ${ }^{3} \mathrm{M}$ Schlesier, ${ }^{3} \mathrm{R}$ Dräger. 'Sapienza University of Rome, Rome, Italy; ${ }^{2}$ University of Freiburg; ${ }^{3}$ Centre of Chronic Immunodeficiency, Freiburg, Germany

CVID are a group of heterogeneous conditions characterized by reduced immunoglobulin levels and absent or poor antibody responses. The latter diagnostic criterion has not been clearly defined leading to a highly variable number and type of immunizations performed among centres. Specific antibody responses cannot be evaluated in patients who are already on immunoglobulin replacement, due to the interference of passively administered IgG. Classification schemes are based on cellular phenotyping and offer instruments for recognition of patients at risk for CVID-associated clinical conditions, but do not take advantage of the possibility to evaluate in vivo antibody responses as a prognostic marker for infectious complications. We immunized 91 CVID patients with a 23-valent pneumococcal polysaccharide vaccine (Pneumovax ${ }^{\circledR}$ ) and measured the IgM and IgA to single pneumococcal polysaccharides before vaccination and 4 weeks later. Results were compared with those obtained using a new IgM and IgA anti-pneumococcal polysaccharides 23-valent assay (PC23). We demonstrated that the IgM/ IgA response to PC23 allows stratifying CVID patients into groups with different risk to experience pneumonia and to develop bronchiectasis. Immunological IgM/IgA responders had the lowest risk for pneumonia $(0 \%)$ and bronchiectasis $(1.2 \%)$, while non responders had the highest risk (37\% and $41.5 \%$ respectively) and IgM-only responders had an intermediate risk (8.8\% and $8 \%$ respectively). The antibody response correlated with the frequency of IgMpos and switched memory B cells. The IgM and IgA PC23 assay represents a valuable prognostic tool for CVID patients and allows investigating the residual antibody production capacity, even in patients on substitutive immunoglobulin replacement.

\section{A DOUBLE BLIND, RANDOMIZED CONTROLLED TRIAL ON THE RESUSCITATION OF PRETERM INFANTS WITH $30 \%$ VERSUS 65\% OXYGEN AT BIRTH}

doi:10.1136/archdischild-2012-302724.0385

'D Rook, ${ }^{1} \mathrm{H}$ Vlaardingerbroek, ${ }^{1,2} \mathrm{H}$ Schierbeek, ${ }^{1,3} \mathrm{AC}$ van der Eijk, ${ }^{4} \mathrm{~J}$ Escobar, ${ }^{5} \mathrm{M}$ Longini, ${ }^{5} \mathrm{G}$ Buonocore, ${ }^{6} \mathrm{M}$ Vento, ${ }^{2,7} \mathrm{JB}$ van Goudoever, ${ }^{1} \mathrm{MJ}$ Vermeulen. 'Pediatrics, Division of Neonatology, Erasmus MC - Sophia Children's Hospital, Rotterdam; ${ }^{2}$ Pediatrics, Academic Medical Center - Emma Children's Hospital, Amsterdam; ${ }^{3 B i o M e c h a n i c a l ~}$ Engineering, Faculty of Mechanical, Maritime \& Materials Engineering, Delft University of Technology, Delft, The Netherlands; 'Neonatal Research Unit, Health Research Institute La Fe, Valencia, Spain; ${ }^{5}$ Pediatrics, Obstetrics and Reproductive Medicine, University of Siena, Siena, Italy; ${ }^{6}$ Pediatrics, Division of Neonatology, University Children's Hospital La Fe, Valencia, Spain; 'Pediatrics, VU University Medical Center, Amsterdam, The Netherlands

Background Resuscitation of term infants at birth with $100 \%$ oxygen increases oxidative stress with concomitant deleterious effects. Optimal levels for preterm infants are unknown. We hypothesized that resuscitation of preterms with initial $\mathrm{FiO}_{2}$ of $30 \%$ is safe, decreases oxidative stress and improves outcome compared to an initial $\mathrm{FiO}_{2}$ of $65 \%$.

Design Preterm infants (GA<32 weeks) were randomized to start resuscitation after birth with $30 \%$ or $65 \%$ oxygen. $\mathrm{FiO}_{2}$ was adjusted based on oxygen saturation and heart rate. Primary outcome was survival without bronchopulmonary dysplasia (BPD) at 36 weeks postmenstrual age. Oxidative stress was determined by urinary DNA and protein oxidation markers, and plasma non protein bound iron.
Results We included 194 infants, mean GA $\left(28^{4} /{ }_{7} \pm 21 / 7\right.$ weeks $)$ and birth weight (1076 \pm 347 gram) were not different between groups. $\mathrm{FiO}_{2}$ was significantly different during the first 5 minutes following birth. Clinical outcomes (table) and oxidative stress markers were not statistically different between groups.

\section{Abstract 385 Table 1 Clinical outcome}

\begin{tabular}{|c|c|c|}
\hline $\mathrm{FiO}_{2}$ & $30 \%(n=99)$ & $65 \%(n=95)$ \\
\hline Mortality (\%) & 6.1 & 10.5 \\
\hline BPD (\%) & 23 & 15 \\
\hline Survival without BPD $(\%)$ & 72 & 75 \\
\hline Intraventricular hemorrhage $\geq$ stage $2(\%)$ & 8.1 & 10.5 \\
\hline Retinopathy of prematurity $\geq$ stage $2(\%)$ & 6.1 & 5.3 \\
\hline Necrotizing enterocolitis $\geq$ stage 2 (\%) & 4.0 & 3.2 \\
\hline
\end{tabular}

Conclusion Resuscitation of preterm infants at birth with $30 \%$ oxygen is as safe as resuscitation with $65 \%$, but does not offer benefits with regard to survival without BPD.

\section{A COUNT OF THREE NEONATAL MORBIDITIES MAY SUBSTITUTE FOR LONG-TERM NEURODEVELOPMENTAL FOLLOW-UP IN VERY LOW BIRTH WEIGHT (VLBW) INFANTS}

doi:10.1136/archdischild-2012-302724.0386

${ }^{1,2} \mathrm{~B}$ Schmidt, ${ }^{2} \mathrm{R}$ Roberts, ${ }^{3 \mathrm{P}}$ Davis, ${ }^{3} \mathrm{~L}$ Doyle, ${ }^{4} \mathrm{E}$ Asztalos, ${ }^{5} \mathrm{G}$ Opie, ${ }^{6} \mathrm{~A}$ Bairam, ${ }^{7} \mathrm{~A}$ Solimano, ${ }^{8} \mathrm{~S}$ Arnon, ${ }^{9} \mathrm{R}$ Sauve, for the Caffeine for Apnea of Prematurity (CAP) Trial Investigators. 'University of Pennsylvania, Philadelphia, PA, USA; ${ }^{2}$ McMaster University, Hamilton, ON, Canada; ${ }^{3}$ University of Melbourne, Melbourne, VIC, Australia; ${ }^{4}$ University of Toronto, Toronto, ON, Canada; ${ }^{5}$ Mercy Hospital, Melbourne, VIC, Australia; ${ }^{6}$ Laval University, Quebec, OC, ' University of British Columbia, Vancouver, BC, Canada; ${ }^{8}$ Meir Medical Centre, Kfar Saba, Israel; ' University of Calgary, Calgary, AB, Canada

Background In very preterm infants who survive to a postmenstrual age (PMA) of 36 weeks, a count of BPD, brain injury and severe ROP predicts the risk of a later death or neurosensory impairment at 18 months (JAMA 2003; 289:1124).

Objective To validate this count of 3 neonatal morbidities as a predictor of poor long-term outcome in VLBW infants who participated in the CAP Trial.

Methods Five-year follow-up of 1514 CAP trial participants who survived to a PMA of 36 weeks. Poor outcome was a late death or survival with one or more disabilities.

Results The incidences of BPD, brain injury and severe ROP were $40 \%, 13 \%$, and $6.0 \%$, respectively. Each morbidity was similarly and independently correlated with a poor 5-year outcome. Table 1 shows the risks of a poor long-term outcome with none, any 1 , any 2 , and all 3 neonatal morbidities.

\section{Abstract 386 Table 1}

\begin{tabular}{llll}
\hline Neonatal morbidities & No. of Infants & $\begin{array}{l}\text { Poor Outcome } \\
\text { at 5 Years }\end{array}$ & $\mathbf{9 5 \%} \mathbf{~ C ~}$ \\
\hline None & 759 & $11 \%$ & 9 to $14 \%$ \\
Any Single Morbidity & 590 & $23 \%$ & 20 to $27 \%$ \\
Any 2 Morbidities & 139 & $44 \%$ & 36 to $53 \%$ \\
All 3 Morbidities & 26 & $62 \%$ & 41 to $80 \%$ \\
\hline
\end{tabular}

Conclusions In VLBW infants who survive to a PMA of 36 weeks, a count of BPD, brain injury and severe ROP predicts the risk of a later death or survival with disability at age 5 years. This morbidity count may substitute for long-term outcome assessments in very preterm infants whose families do not comply with neurodevelopmental follow up. 